\title{
The Mobile Game Design about Chinese Character Modules for Cultivating Chinese Character Thought
}

\author{
YanWang \\ Harbin Institute of Technology \\ HIT \\ Harbin, China \\ wy0126@126.com
}

\author{
XinGuo \\ Harbin Institute of Technology \\ HIT \\ Harbin, China \\ gxcindy93@163.com
}

\author{
TongChen \\ Harbin Institute of Technology \\ HIT \\ Harbin, China \\ tonghit@126.com
}

\begin{abstract}
With the decline of Chinese character thought and Chinese language learning craze, we hope to help Chinese learners cultivate Chinese character thought imperceptibly and improve the cognition efficiency of Chinese characters on entertainment. On the basis of principles of Chinese characters and the experimental Cognitive results from Psychologist, we put forward a design method about Chinese character modules. Modules combinations decide on the location of modules. We present the partition design to retrieve the location of modules. Than measuring the results of the module combination by preset value will provide immediate feedback to users. In order to illustrate the feasibility of the method, we develop the Chinese characters game in smart mobile terminal which in the form of riddle to enhance entertainment. We expect that the method will explore a new type of digital mobile learning in Chinese subject construction. Intensive study about partition design will make more Chinese characters applicable on this system.
\end{abstract}

Keywords-Chinese character thought; Chinese character modules; Chinese culture education; game design; M-learning; Chinese subject construction

\section{INTRODUCTION}

Chinese characters, a bright pearl of Chinese culture, have been the longest continuously. Chinese characters have decisive effect on the Chinese traditional thought pattern that different from English letters linear thought pattern. The Chinese traditional thought pattern is a kind of "intuition thinking schema[1]."Image thinking" system is the unique feature of Chinese character thought. However, under the background of globalization and digitalization, Chinese characters and Chinese character thought encountered great challenge.

\section{A. Global cultural convergence and The weakening of the differences in linguistic thinking}

Information technology revolution has a revolutionary effect on the Chinese, especially in words and using grammar. Since the late 20th Century, English learning begins to roll up in Chinese mainland. Chinese students pay a lot of effort and time on English learning, much more than Chinese learning[2].Young people have grown accustomed to using new linguistic thinking, like "pidgin English"(a protoChinglish term).It is no exaggeration to say that contemporary youth appears to be retreating from Chinese character thought.
B. Digital information revolution and the weakening of the Chinese character thought

With the advent of the computer age, Chinese characters are confronting a crisis. The way to input English characters to computers entirely harmonious with qwerty keyboard. But Chinese character input faces a great conundrum. Nowadays the Pinyin input method that can associate with words become the most popular and common input method in China. It makes completely do not need to understand the basic configuration of Chinese characters. Users can output correct Chinese words do not need to input characters completely, even misspelled. Much convenience was brought to Users. At the same time, Chinese characters and Chinese character thought was deeply damaged by the Pinyin input method.

We randomly send a questionnaire survey about input method to 66 college students. The results were astonishing: $100 \%$ of college students use pinyin input method, $14 \%$ of respondents admitted often having problems writing characters. Nearly $80 \%$ of respondents occasionally having problems on writing[3].It reflects the digital Chinese characters input method have significant effects on young people in Chinese character thought. According to the data of 12 cities such as Beijing, Shanghai and Guangzhou about "Chinese writing habit" from a domestic well-known mass observation. 94.1\%percent of the respondents admitted having problems writing characters. $26.8 \%$ of them often having writing problems[4].

Despite all this, we find the digital Information Technology also provide a new concept to digital processing of Chinese characters. On the one hand, we should develop Chinese style computer tools suitable for Chinese characters input according to the hardware transformation, the development focuses on Chinese character's forms and meanings by means of current hardware. The combination of Chinese characters input with literacy and teaching is very helpful in building students good education foundation. The government should strengthen their management on the writing of Chinese characters. [5] On the other hand, we have to seize this education moment to use digital technology, especially of intelligent mobile terminal, develop new methods of learning Chinese characters for young people.

This paper tries to use Chinese character modules to design the Chinese characters game in smart mobile terminal. Through 
entertaining and educational way to help Chinese learners cultivate Chinese character thought imperceptibly and explore a new type of digital mobile learning in Chinese subject construction.

\section{THE PROJECT METHODOLOGY AND GUIDELINES}

\section{A. Characterization of Chinese characters.}

Seen from Chinese characters structure pattern, Chinese characters are seen as square framework, which includes top, bottom, left and right of all space. Secondly, they are full with modules. Modular thought is the corporate wisdom of mankind. Chinese characters modules feature four basic characteristics as follows:

- Regularity Chinese characters modules breaks down into three basic structures: up-low structure, left-right structure, hybrid structure (surrounded structure and surrounded by half structure). The uniqueness of individual Chinese characters is that it depends on the selection, the number and the position of the module.

- Substitutability Different characters can contain the same module; the module has the same position and works in the same way in the construction principles of Chinese. For example, “河” and “江” have the same module - “ $; ”$, this module located in left part of characters and represents the character relevant to water. If replace “可” the right part of “河” to “工”, the “河” will be change to “江” .

- Relative fixity Modules have freedom when they formed the whole word. However, the combination mode is not random; it is bound by some rules and conditions that limit the possible of arrangement mode. The spelling directions of modules are never changed [5]. The positions of the most modules are fixed, especially the module which cannot be the single character. For example, “个” appears on the left-hand part of characters. "\#” appears on the top-hand part of characters.

- Hierarchy Complex modules can contain other simpler modules. For example, “言” as one module of “警” , at the same time, it also contain simple modules, like “口”.

\begin{tabular}{|c|c|c|}
\hline 字型结构 & 示童国 & 宇俾 \\
\hline 左右 & $\begin{array}{ll}\text { II III } \\
\text { I: }\end{array}$ & $\begin{array}{ll}\text { 他 } & \text { 湖 } \\
\text { 侣封 }\end{array}$ \\
\hline 上下 & $\begin{array}{l}=\equiv \\
\text { I }\end{array}$ & $\begin{array}{ll} & \text { 呆 } \\
\text { 品 } & \text { r }\end{array}$ \\
\hline 旅合 & 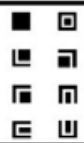 & 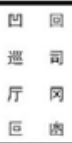 \\
\hline
\end{tabular}

Fig. 1. Chinese characters structure diagram

Chinese characters modules are limited, but the module combination mode can build more than 90000 characters. Chinese characters composed of modules justice to the spatial image thinking. The thinking is an important structural characteristic of Chinese characters shape code and an obvious distinction of characters which have two-dimensional space and alphabetic writing which laid out the one-dimensional linear[6]. Chinese characters which framed by space, united by modules and cored by image are a special symbol of Chinese civilization. The formation of Chinese characters is that Yin and Yang are moving, interacting each other. It always dominated the Chinese worldview and formed a unique Chinese character thought.

\section{B. The formation of Chinese characters thought}

At first, the research of psychology shows that orthographic awareness is not so much depends on Chinese character component structure, but rather depends on the space location of the Chinese characters. Stated more narrowly, It is dependent on how closely the module to the whole consciousness of glyphs[7]. Based on the two-dimensional space of the mobile terminal, students piece together characters to keep it in mind. The method facilitates the students' memorization and help them mastery of characters.

In the current period of education development, the teaching way "Chinese characters module split method" accepted by Chinese educators and front-line teachers who are putting it into practice. According to the statistics made by professor LuoWeidong, teacher of Beijing Language and Culture University, $90 \%$ of the Chinese teachers who gives the courses for foreigners used module teaching method in the teaching process. Explaining the roles played in characters of module can help students understand the relationship between graphic, phonological and semantic properly and increase capacity to correctly written characters. It can also tell students formation laws of Chinese character and help them build a systematic and scientific Chinese character configuration thought[8].the data does shed revealing light on the fact that using module to learn and remember characters conforms to the rules of language teaching for second language learners, help them focus on the gradual progress that is from elementary to profound.

\section{THE PRINCIPLES OF CHINESE CHARACTERS MODULES GAME DESIGN}

We can use Chinese modules and the law of formation to design the Chinese characters modules game. Doing puzzles will increase entertainment of the game. The users can understand the laws of formation and cultivate Chinese character thought by piece all characters modules.

\section{A. The principle of section of font}

Selection appropriate modules for characters piecing and evaluate the correctness of result is the regulation of characters modules game. So the section of whole word and establishment of the modules database are crucial. The game is speculated that it would be better for primary Chinese characters, like primary 1 to 5 Chinese pupils and non-native students with intermediate Chinese who have learned Chinese for more than one year. The standard vocabulary of Chinese curriculum in 2001 about different learning periods shows: first-graders pupils should recognize1600-1800 characters and master 800-1000 characters[9].In 2002, The Office of Chinese Language 
Teaching Abroad leased "Chinese teaching outline for foreign students of university" stipulate that there are 1414 Chinese characters on the initial stage of Chinese learning,700Chinese characters on the intermediate stage of Chinese learning, 451Chinese characters on the higher stage of Chinese learning[10]. There are around 1500 Chinese characters with higher frequency which are useful to improve the ability of Chinese characters reading .Finding overlapping Chinese characters through filters three data example, classifying characters by module's type, sorting the modules of characters in descending order. Then choose Chinese characters modules with higher frequency into font library.

\section{B. The principle of game Levels}

The game is in the form of riddle. Therefore, the structure and complexity degree of Chinese characters formation determines how difficult the process will be.

The degree of levels is based on the complex and briefness of Chinese characters modules combination. Yu Bolin, a famous psychologist in China, proved that people identify different structure with different time, showing that up-down structure is more difficult than left-right structure. Therefore, the first level is left-right structure and the second is up-down structure. From Chinese characters modules formation mode diagram, we can see that some left-right structure characters exist in up-down structure and vice versa. Because of complex structure, this kind of characters is put into the third level. The fourth level should select hybrid structure characters, including sole character and surrounding and half of surrounding structure characters. This kind of characters has a complex structure and is difficult to understand from their structure, therefore, they are put into the highest level. The difficulty of four levels is rising gradually, increasing the challenge of this game.

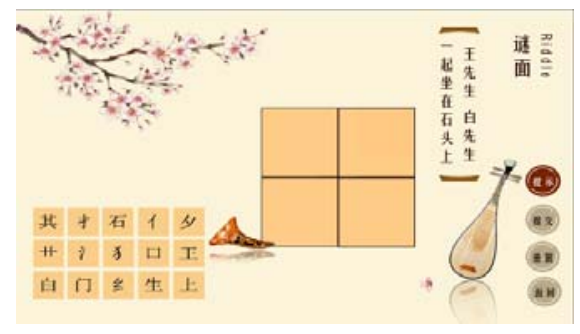

Fig. 2. The interface of game level 1

\section{Choice of the Mobile Platform}

This design is used on the mobile terminal. With the change of mobile screen and promotion of mobile application experience, the mobile phone has become the most popular Internet terminal. Meanwhile, mobile devices will be the main stream of education application in the future. The future education will have more new application skills and communication ways to serve mobile study and create highly intelligent study environment. Through great convenience of mobile study, users can learn Chinese characters by this character module game to change passive learning state and use game to learn Chinese characters.

\section{Key TeChnology of Chinese Character Modules GAME}

\section{A. Partition design}

The partition design of operating area is the basic link in this module game. Modules combinations decide on the location of module, and every location is fixed. According to the variability of module form, modules have different proportion change in different locations to realize the whole harmony. In accordance with the principle above, we divide Tin Word format into nine small areas to satisfy the Chinese characters needs of up-down structure, left-right structure, “品” structure and inverted “品” structure. If you want to meet all of the Chinese characters formation, you should further divide in nine areas, that is to say, partition can limit the placement of the module and proportion.

The main way of Chinese character module game is dragging game modules into Tin Word format. In this process, we should realize haul and placement of Chinese characters. We can add mesh collider to the module, which can have interaction with the Chinese characters modules through the single point touch. We can definite module of screen coordinates and spatial coordinates of the game, click and drag the module, and transfer game screen coordinates into space coordinates, then calculate the offset and determine space coordinates position after moving modules to implement drag and movement of modules.
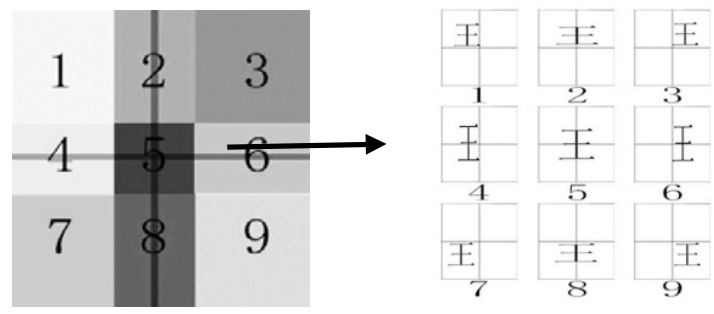

Fig. 3. Partition diagram of nine grids and the position of every part in Chinese character “王” and their proportions.

Firstly, we can amplifier module picture for $1 / 2$ of the proportion of the format when we put characters in Tin Word formats, then judge module make collision with which partition and update location and scale of module, making it easy for users to choose the final placement. The location of the module in each partition and proportion as shown in figure 2 .

\section{B. True or False}

After submitting module combination, then measure the results of the module combination. This level answer is certain in practice case, so presetting partition information according to the answers. After users submitting the answer, recording variable information in every partition, compared with the preset information traversing all partitions. If the answer is exactly right, users could enter into video interface to further study, or vice versa if combination is wrong, users would fail, and every part should be combined again. Take “碧” as an example, in Tin Word format, this character will take three positions which are left-up, right-up and down, corresponding positions of No.1, No.3 and No.8. In these positions, we can 
preset Chinese character modules, and No.1 is “王” , No.3 is “白” and No.8 is “石”. After submitting, the system will compare the module information of No.1, No.3 and No.8, and users will win the game when three modules corresponding at the same time.

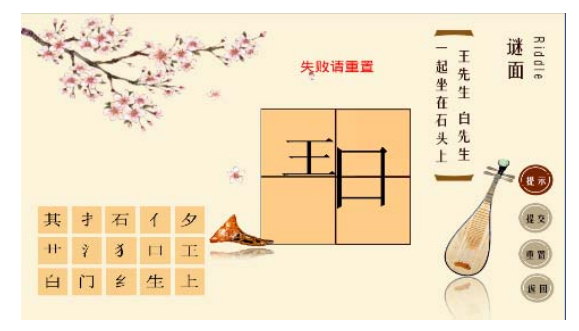

Fig. 4. The interface of game failure

\section{Practice results}

Clicking the Logo at the mobile intelligent terminal interface into the game, there are three main buttons on the game interface connecting music control interface respectively to control music playback, checkpoint choice interface and the game introduction interface.

After entering level interface, according to the riddle guessing the corresponding Chinese characters, if users could not guess the riddle, they could click the tips button to get prompt and save the users' time. Dragging and the characters into operation area, if they were wrong, operations could be reset, submitting after splitting right. Then, users can enter the interface of interpretation, playing the Chinese characters' introduction videos, from the aspects of pronunciation, configuration, and meaning explanation, which help users understand the Chinese characters. If they were wrong, prompting the user to reset the game. After experiencing all levels, users can return the main interface.

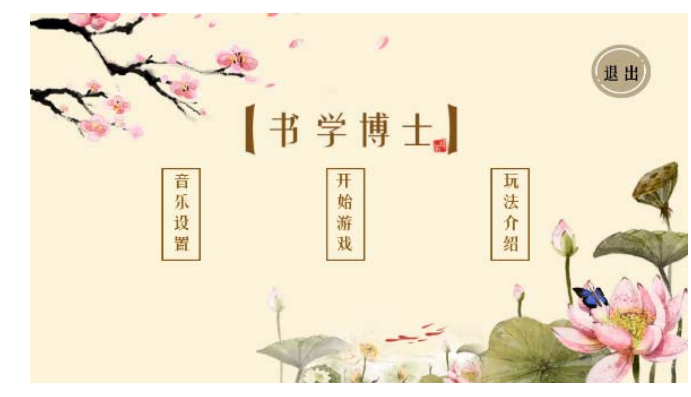

Fig. 5. The main interface of game

\section{CONCLUSION}

In this paper we have highlighted the great effect of Chinese character thought on Chinese science education. This innovation of this design research is that Chinese character game abandons traditional mobile Chinese characters game, in combination with the configuration of Chinese characters, integrating Chinese character modules to research. The Chinese character module game based on mobile platform combines module teaching and mobile learning, paying attention to the user's initiative and enthusiasm. Combining education with recreation can help Chinese learners' consciousness of Chinese character formation and learn Chinese characters by themselves to promote the digital heritage of traditional Chinese culture, which is an innovative way of learning Chinese characters.

What's more, designing and developing games can use mobile platform. The mobile platform is a media with a broad prospect. Mcluhan once said, "Medium is the information." The Chinese character module game makes users to regain his childhood in the entertainment through the new technology development platform to set up an auxiliary means of Chinese character thought.

\section{ACKNOWLEDGMENT}

We gratefully acknowledge the fund support of national social science fund project "Research on the Basic Problem of Literature and Art in the Technology of Phenomenological Horizon" (project number:12BZW017) achievements.

\section{REFERENCES}

[1] Shihu, "The discussion about Chinese characters thought," Poetry Exploration, vol. 2, pp. 529-551, 1996.(In Chinese)

[2] RenXueli, "Security characters, maintain native language and carry forward the Chinese traditional culture," Journal of Socialist Theory Guide, vol.11, pp. 118-119, 2006.(In Chinese)

[3] Original source of data:http://www.sojump.com/report/6602558.aspx?qc=.

[4] QingBo, “The crisis of Chinese characters?" Youth exhibition, vol.1, pp. 4-5, 2006.(In Chinese)

[5] WangWei, "Several strategies to strengthen the Chinese characters handwritten during the generation of computers," Journal of Zhoukou Normal University,vol. 4, pp. 75-78, 2009.(In Chinese)

[6] Ledderose, "Ten Thousand Things: Module and Mass Production in Chinese Art," Zhangzong, Translation. BeiJing:SDX Joint Publishing Company, pp. 23, 2006.

[7] YuBolin. CaoHeqi, "The cognition of Characters structure,". The study in psychological science, vol.5, pp. 5-10, 1992.(In Chinese)

[8] YuBolin, CaoHeqi, FengLing, LiWenlin. "The influence from Chinese characters morphological code and phonetic code to parts recognition." Acta Psychologica Sinica, vol.3, pp.232-239, 1990.(In Chinese)

[9] WangMin, "The investigation about chinese handwriting education in primary school," Applied Linguistics III, pp.232-239, 2014.(In Chinese)

[10] HuGenlin, TaoBenyi, "Chinese teaching outline (curriculum standards) primary school literacy quantity," Education scientific research, vol.3, pp.51-55, 2009.(In Chinese)

[11] The Office of Chinese Language Teaching Abroad. Chinese teaching outline to the HSK outline in college. Beijing Language and Culture University Press,2002.(In Chinese) 